\title{
WINDS AND MASS-LOSS FROM EVOLVED, LOW-GRAVITY COOL STARS
}

\author{
K. G. CARPENTER \\ Laboratory for Astronomy and Solar Physics, NASA-GSFC, USA \\ hrscarpenter@tma1.gsfc.nasa.gov
}

AND

R. D. ROBINSON

Astronomy Programs, Computer Sciences Corporation, @NASA-GSFC, USA

hrsrobinson@stars.gsfc.nasa.gov

The character of the winds and the amount of mass-loss from stars during the red giant and supergiant stage of evolution are critical to the progression of that evolution. We therefore summarize here the results of a series of programs utilizing the Goddard High Resolution Spectrograph (GHRS) on the Hubble Space Telescope (HST) to study the winds and mass-loss from the surface of evolved, low-gravity cool stars. We discuss the structure of the chromospheres in which the base of these stellar winds lie, the velocity of the wind and its acceleration with height, and what these spectra tell us about the mass-loss from these stars. Because of its relevance to this Joint Discussion, we will also discuss in some detail what we have learned about real-time variability of these phenomena and the implied somewhat erratic nature of the mass-loss process in at least some of these stars. 